\title{
On the Performance of Increasing Subcarrier Order in Multi-Band Carrier-less Amplitude and Phase Modulation for Short Haul Optical Links
}

\author{
Paul Anthony Haigh ${ }^{1}$, Jinlong Wei ${ }^{2}$, Tu Nguyen ${ }^{3}$ \\ ${ }^{I}$ Communications and Information Systems, University College London, London, WC1E 6BT, UK \\ ${ }^{2}$ Huawei Technologies Duesseldorf GmbH, European Research Center, Germany \\ ${ }^{3}$ University of Mons, Mons, Belgium \\ p.haigh@ucl.ac.uk
}

\begin{abstract}
We show experimentally, for the first time, that an increasing subcarrier order in $m$ CAP optical-links does not result in proportional data-rate gain. We show that 10-CAP offers similar performance to 20-CAP with substantially less complexity.
\end{abstract}

OCIS codes: $060.4080,060.2330$.

\section{Introduction}

Multi-band carrier-less amplitude and phase modulation ( $m$-CAP) is becoming prevalent across optical communications with particular focus on short haul links, with numerous recent reports in the literature [1-4]. In [1], the $m$-CAP concept was introduced and experimentally demonstrated. It was shown that by splitting a bandwidth into $m$ subbands, or subcarriers for consistency with OFDM nomenclature, several advantages occur such as improved tolerance to chromatic dispersion and improved sensitivity in a band-limited environment with no loss of data rate; 1-CAP could support $100 \mathrm{~Gb} / \mathrm{s}$, while $102.4 \mathrm{~Gb} / \mathrm{s}$ is supported with 6-CAP. This work was further extended and developed, with a recent reports published in $[4,5]$ showing high speed multi-Gb/s $m$-CAP links with $m=12$ and 10 subcarriers, respectively. From the reports in the literature, it is abundantly clear that high speed links can be supported using such a multi-band scheme which also allows the introduction of bit- and power-loading algorithms. However, one major impediment in the progress of this scheme is computational complexity, since costly finite impulse response (FIR) filters are used to generate the pulse shaping filters inherent in $m$-CAP links [1]. It is well known that the computational complexity of the system at the transmitter is $2 L /$ symbol, where $L$ is the filter length and the 2 is present because there are both an in-phase and quadrature FIR filter present. As such, the number of operations required increases linearly with $m$ while simultaneously the processing area available in real time systems has limitations. Therefore, considering that the order of $m$ in the various literature reports appear to be arbitrarily selected, an investigation into the capacity improvement as a function of $m$ is required. We experimentally show here, for the first time, that increasing $m$ does not linearly improve the measured data rate after bit-loading, and instead and exponential relationship is inferred, with a horizontal asymptote. We show that for $m=\{1,2,5,10,15,20\}$, data rates of $\sim 16.67,16.67,20,23.33,24.44$ and $25 \mathrm{~Gb} / \mathrm{s}$ can be achieved, respectively, and hence, the ratio of improvement in data rate actually decreases with increasing $m$, until a limit is reached.

\section{Test Setup}

A schematic block diagram of the system under test is shown in Fig. 1. Firstly, $m$ independent pseudorandom datasets $\left\{d_{1}, \ldots, d_{m}\right\}$ of length $10^{5}$ are generated in MATLAB and mapped into the binary phase shift keying (BPSK) modulation format (i.e. $\left\{k_{1}, \ldots, k_{m}\right\}=1$ ) up-sampled via zero padding to match the required $m$-CAP sampling frequency [1]. The up-sampled signals are then split into their in-phase and quadrature components before passing through pulse shaping FIR filters whose impulse responses correspond to a Hilbert pair orthogonal in time. The mathematics of the $m$-CAP pulse shaping filters are not shown here or described due to space constraints but can be found in [1]. Next, each component is summed and used to intensity modulate a $194 \mathrm{THz}$ wavelength via a properly biased electro-optic modulator (EOM) with $40 \mathrm{GHz}$ bandwidth. A Tektronix AWG70001A (14 bits, $14 \mathrm{GHz}$ bandwidth) was used as a digital-to-analogue converter and each of the $m$-CAP signals under test are designed with $10 \mathrm{GHz}$ total bandwidth, while the EOM was selected with excess bandwidth in order to ensure that the link was free of a band-limitation at 
the transmitter at the cost of introduction of additional out-of-band noise that is later filtered after analogue-to-digital conversion in MATLAB via a low-pass filter. After polarization control, the signals are transmitted over $40 \mathrm{~km}$ of standard single mode fibre (SSMF) and amplified in order to ensure there was always $0 \mathrm{~dB}$ impinging on the Finisar XPRV2022A photoreceiver (33 GHz, inbuilt transimpedance amplifier) across each $m$-CAP signal under test. The resulting photovoltage is sampled at $80 \mathrm{GHz}$ by a Keysight DSOX93304Q real time oscilloscope ( $8 \mathrm{bits}, 33 \mathrm{GHz}$ ) before time-reversed matched filtering, down-sampling, phase correction, constellation de-mapping and finally estimation of the transmitted bits occurs. Next, in MATLAB, the error vector magnitude (EVM) is measured from the received constellations and the signal-to-noise ratio (SNR) estimated according to [6]. A target bit-error rate (BER) is set to $3.8 \times 10^{-3}$ in keeping with the $7 \%$ overhead forward error correction floor. Following equation (4) in [6], the appropriate constellation cardinality $k$ is selected for each subcarrier across all values of $m$ under test, before the experiment is repeated as outlined previously with the new values of $k$.

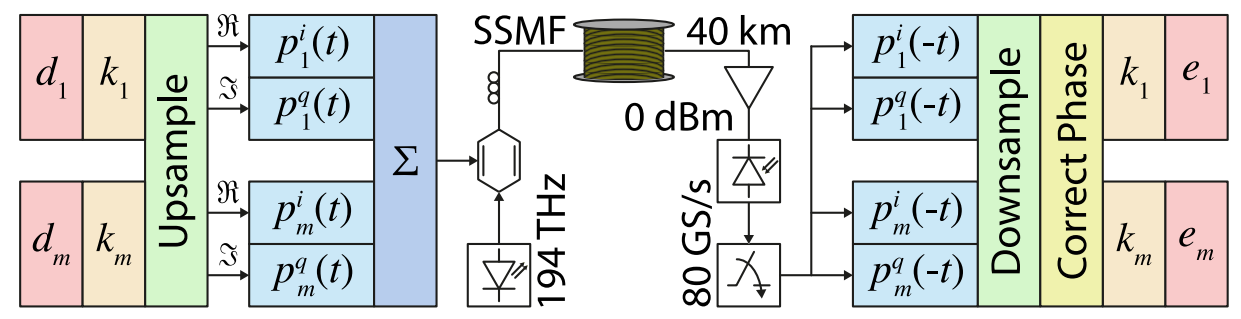

Fig. 1: Schematic block diagram of the proposed test setup investigating $m$-CAP capacity

\section{Results}

The inferred values for $k$ are illustrated in Fig. 2(a), except for $m=1$ which has $k_{1}=2$. It is clear from Fig. 2(a) that as the order of $m$ increases, the number of higher cardinality subcarriers increases. However, what is not clear is how much improvement in capacity this brings. For example, taking $m=20$ as an example in comparison to $m=10$; the subcarrier baud rate for $m=20, R_{s 20}$, is $\sim 417 \mathrm{MBd}$, following $R_{s}=m^{-1} B(1+\beta)^{-1}$, where $B$ is the total signal bandwidth and $\beta$ is the pulse shaping roll-off factor, set to 0.2 in this case as is consistent with the literature [1]. For $m=10, R_{s 10}$ is $\sim 833 \mathrm{MBd}$, which is approximately double, as expected, since the subcarriers are set with even bandwidths. Once more considering $m=20$, this means that if we set two subcarriers at $k=4$ and the rest to $k=0$, and one subcarrier for $m=10$ to $k=4$ and the rest to $k=0$, the obtained transmission speeds will be identical in both cases. In this case, based on reports in the literature, we would still expect $m=20$ to offer marginally improved EVM/BER performance than $m=10$ over short haul fibre links due to the fact that it offers improved bandwidth sensitivity and tolerance to chromatic dispersion. Simultaneously, one must consider whether the added number of operations present in the transmitter and receiver digital signal processors is worth the marginally improved performance, since $m=20$ introduces twice the number of operations-per-symbol than $m=10$. It should be noted that in this system no subcarriers in any order of $m$ tested required a constellation size beyond $k=4$, as non-square constellation shapes such as $k=3$ and 5 were not considered in this work and there was not enough SNR present in any subcarrier for $k \geq 6$.

The BER performance was measured in MATLAB in a symbol-by-symbol manner and is presented in Fig. 2(b) for $m=\{20,15,10,5\}$. The BER performance of $m=2$ and $m=1$ are not included in Fig. 2(b) due to space constraints, however, BERs of $10^{-5}$ where obtained for both subcarriers in the $m=2$ configuration which is better than expected. The reason for this is that the measured SNRs for $m=2$ were insufficient for $k=4$, however were enough for $k=3$, which was not utilized in this work. For $m=1$, a BER of $\sim 2.1 \times 10^{-4}$ was measured. For the subcarriers displayed in Fig. 2(b), all of the measured BERs meet the $3.8 \times 10^{-3}$ target. Interestingly, a pattern emerges for these subcarriers, as can be seen inset, where the $x$-axis in each case has been normalized by a factor of $m$. All of the BER profiles for each order of $m$ follow the same trend where the BER performance degrades with increasing sub-band carrier frequency, which can be attributed to the decreasing SNR profile of the links as is reflected in Fig. 2(a).

Fig. 2(c) shows the total measured data rate for each order of $m$. It is immediately obvious that the increase in available transmission speed is not linearly proportional to $m$, but is in fact an exponential relationship as predicted. The curve is fit with the equation inset in Fig. 2(c) which predicts that an asymptotic data rate is reached at approximately $25.5 \mathrm{~Gb} / \mathrm{s}$ for this particular system. The number of operations is also shown on the right $y$-axis, showing a linearly increasing relationship with $m$. Now, taking the three highest data rates achieved in this system, i.e. $25,24.44$ and 

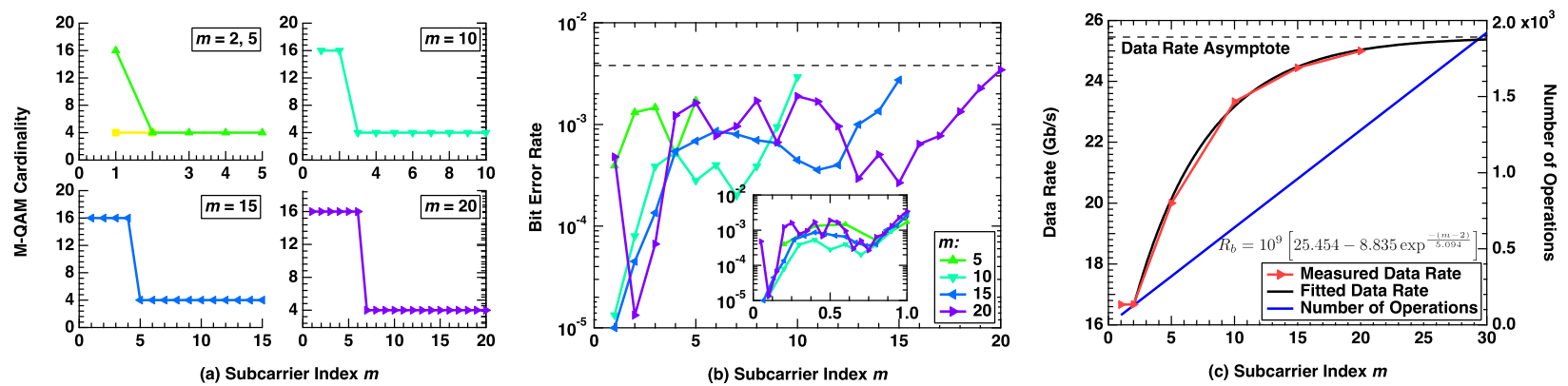

Fig. 2: (a) the assigned modulation cardinalities for each higher order of $m$, (b) the measured BER and (c) the data rate as a function of $m$

$23.33 \mathrm{~Gb} / \mathrm{s}$ which were achieved with $m=20,15$ and 10 , respectively, the corresponding number of computational operations (in the transmitter) are 960, 640 and 320, respectively. Considering the data rate difference between $m=20$ and $m=10$ is $\sim 1.7 \mathrm{~Gb} / \mathrm{s}$, or a net reduction of $4 \%$, the number of operations actually trebles in this time, meaning the DSP area required increases threefold. This result is key, because it enables researchers to thoroughly understand the trade-offs between the number of subcarriers present in their $m$-CAP links and the actual improvement in data rate obtained. This is further supported by the fact there was no clear improvement in BER performance (Fig. 2(b)) or constellation cardinality assignment (Fig. 2(a)). We envisage that this result will ensure proper consideration and therefore design of $m$-CAP links and warrants further investigation into the relative differences in performance in further aspects of optical communications such as chromatic dispersion.

\section{Conclusion}

We have shown, for the first time, that simply increasing the number of subcarriers within multi-band carrier-less amplitude and phase modulation over short haul optical links does not result in a proportional data rate gain. We show that the transmission speed increases to an asymptotic data rate that depends on the SNR present in the system, while the number of computational operations increases linearly with $m$.

\section{Acknowledgements}

This work was supported by the UK Engineering and Physical Sciences Research Council (EPSRC) grant EP/P006280/1: Multifunctional Polymer Light-Emitting Diodes with Visible Light Communications (MARVEL).

\section{References}

1. M. I. Olmedo, et al., "Multiband Carrierless Amplitude Phase Modulation for High Capacity Optical Data Links," in Journal of Lightwave Technology, vol. 32, no. 4, pp. 798-804, Feb.15, 2014.

2. J. Zhang, et al., " $11 \times 5 \times 9.3 \mathrm{~Gb} / \mathrm{s}$ WDM-CAP-PON based on optical single-side band multi-level multi-band carrier-less amplitude and phase modulation with direct detection" Optics express, Optical Society of America, 2013, 21, 18842-18848

3. L. Sun, et al., "Multiband Three-Dimensional Carrierless Amplitude Phase Modulation for Short Reach Optical Communications" Journal of Lightwave Technology, 2016, 34, 3103-3109

4. J. Wei, et al., "56 Gb/s multi-band CAP for data center interconnects up to an $80 \mathrm{~km} \mathrm{SMF"} \mathrm{Opt.} \mathrm{Lett.,} \mathrm{OSA,}$ 2016, 41, 4122-4125

5. R. Puerta, et al., "107.5 Gb/s $850 \mathrm{~nm}$ multi- and single-mode VCSEL transmission over 10 and $100 \mathrm{~m}$ of multimode fiber", Optical Fiber Communication Conference Postdeadline Papers, Optical Society of America, 2016, Th5B.5

6. R. Schmogrow, et al., "Error Vector Magnitude as a Performance Measure for Advanced Modulation Formats", IEEE Photonics Technology Letters, 2012, 24, 61-63 